\title{
Preliminary Design Method FOR BASE BLEED UNIT
}

\section{SERDAREVIC-KADIC, S. \& TERZIC, J.}

Abstract: Base bleed is a system for base drag reduction of projectile by gas injection into base area. The gas injecting through orifice is generated by combustion of solid propellant grain. Base bleed output mass flow rate depends on the chamber pressure, the pressure into which jet exhausts, time and spin rate. An analytical interior ballistics model of base bleed developing in the paper is based on basic fluid dynamics and experimental results of measured time for base bleed burning in the field of radial acceleration. By iterative method, chamber pressure and mass flow rate can be estimated during the base bleed work. The model is verified by available experimental results.

Key words: Bleeding, Chamber, Spin, Pressure, Reduction
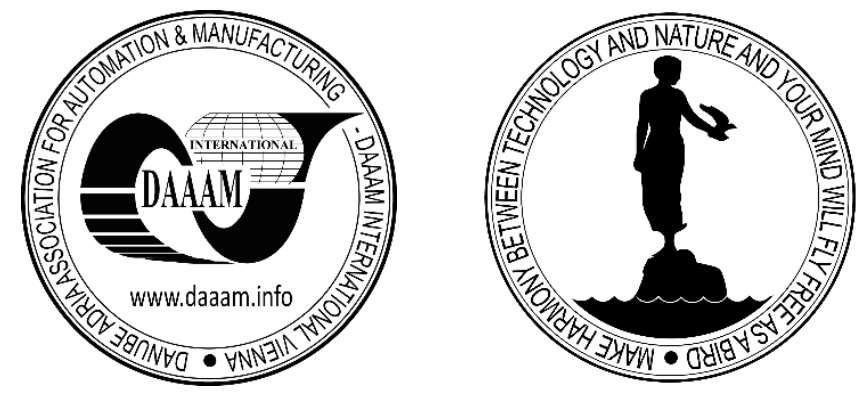

Authors' data: Assist. Prof. Dr. Sc. Serdarevic-Kadic S[abina]; Assist. Prof. Dr. Sc. Terzic, J[asmin], Faculty of Mechanical Engineering, University of Sarajevo, Vilsonovo setaliste 9, Sarajevo 71000, Bosnia and Herzegovina, serdarevic@mef.unsa.ba, terzic@mef.unsa.ba.

This Publication has to be referred as: Serdarevic-Kadic, S[abina] \& Terzic, J[asmin] (2019). Preliminary Design Method for Base Bleed Unit, Chapter 05 in DAAAM International Scientific Book 2019, pp.055-070, B. Katalinic (Ed.), Published by DAAAM International, ISBN 978-3-902734-24-2, ISSN 1726-9687, Vienna, Austria

DOI: $10.2507 /$ daaam.scibook.2019.05 


\section{Introduction}

Except increasing lethal efficiency at target (Zecevic et al., 2015), the basic requirement for designers of projectile is to increase the range. One of the ways that can be used to achieve improved performance of the projectile, in order to increase the range of the projectile, is base drag reducing of the projectile.

Reduction of base drag, it can be higher of $50 \%$ of total drag in transonic regime, is realized by increasing base pressure which can be achieved in either of the following: by base cavity, by aerodynamic optimization of projectile rear portion or by base bleed application.

During projectile flight, region of underpressure is created behind the base of the projectile. The region leads to curve of streamlines to axis. Close to axis, streamlines have to curve again to be parallel with axis. The phenomenon is followed with shock waves and vortex. At the point of streamlines impact, overpressure zone is made and air is flowing from the zone to base of projectile and is filling vacuum on the base. Curved boundary layer extension pulls air from base of projectile. On this way recirculation region is made behind the base (Fig. 1.).

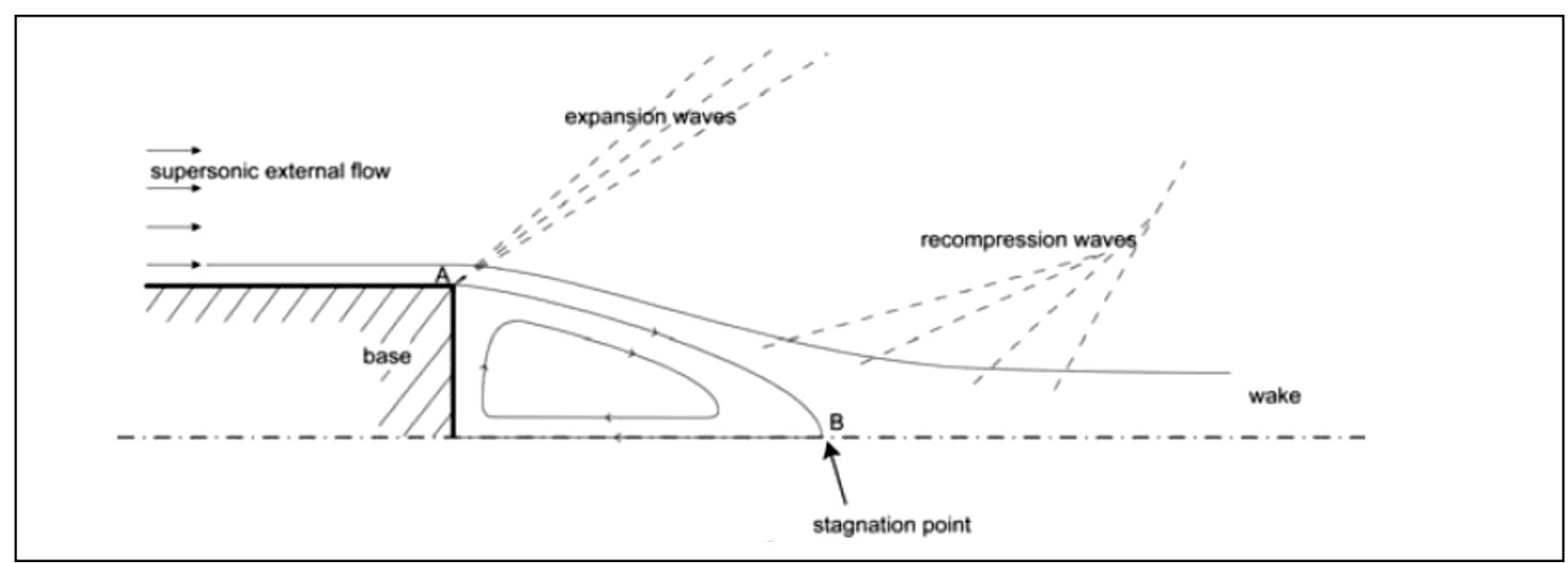

Fig. 1. Flow field behind a projectile without base bleed unit

Injecting small amounts of gas into the flow field behind the base of the projectile (Fig. 2.) will split the originally large recirculation zone into two halves - one recirculation region remains at the symmetry axis (PRR - primary recirculation region), and the other one is formed right behind the base corner (SRR - secondary recirculation region).

As the mass flow rate is increased, the recirculation zone at the axis is pushed further out, and the other one at the base corner becomes larger. If the mass flow rate is increased away, the recirculation region near the axis disappears, and the base-bleed flow follows a straight path.

Base bleed is system of reducing base drag by injection gas generated by burning composite propellant into the base area. 


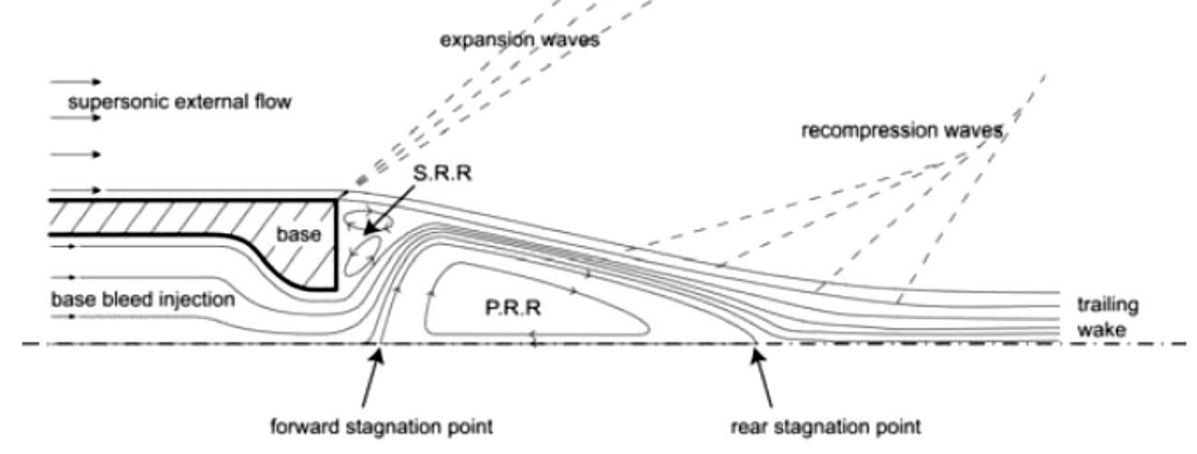

Fig. 2. Flow field behind a projectile with base bleed unit

Effects of gas injection into the base area of projectile were a subject of experimental (Baker et al., 1951, Murthy et al., 1976, Murthy \& Osborn, 1976, Strahle et al. 1982., Kayser et al., 1988) and numerical (Sahu et al., 1985, Sahu, 1986) studies (Serdarevic-Kadic et al., 2012). Extensive experimental investigations on projectile $155 \mathrm{~mm}, \mathrm{M} 864$, were made during the last decades of the twentieth century. The time of base bleed burning in the function of projectile spin during ground testing were measured (Kayser et al., 1988) and they were measured pressure and temperature in base region during the projectile flight. The results of the experiments were applied to develop an engineering model for the flight performance of the M864 base bleed projectile computation (Danberg, 1990).

The solid propellant combustion (Nietubicz\&Gibeling, 1995) was modeled and the results are incorporated to Navier-Stokes code to simulate flow field in the base region of M864 projectile. Eddy breakup model for chemical reactions is implemented to FINFLO solver and indicated the dramatic effect which chemical reactions have on the aerodynamic drag of a base bleed projectile (Kaurinkoski, 2000).

There are significant numbers of reports about extended range projectile by base bleed, based on theoretical, experimental and numerical methods (Belaidouni, 2016), but there are mainly concentrated to trajectory estimating.

The resistance of air is the largest at ascending branch of trajectory, particularly in the initial part when velocity of projectile rapidly decreases, so effect of base bleed unit is the largest in this regime. The primary purpose of this study is to develop a model for quick design of base bleed units basing on propellant type, geometrical characteristics of grain and spin rate. The secondary objective is to determine how the number of grain segments affects to working time of base bleed unit for different grain shapes.

\section{Physical model of gas generation}

Base bleed unit is intended for increasing the range of a projectile with usage a concept of base drag reduction of the projectile. Reducing the base drag of projectile is accomplished by filling space behind the projectile base with combustion products to increase pressure to the value close to the ambient pressure. 
Products of combustion which are bleeding through the orifice in the projectile base are produced by combustion of grain (shaped propellant charge, Fig. 3.) in the combustion chamber of base bleed.

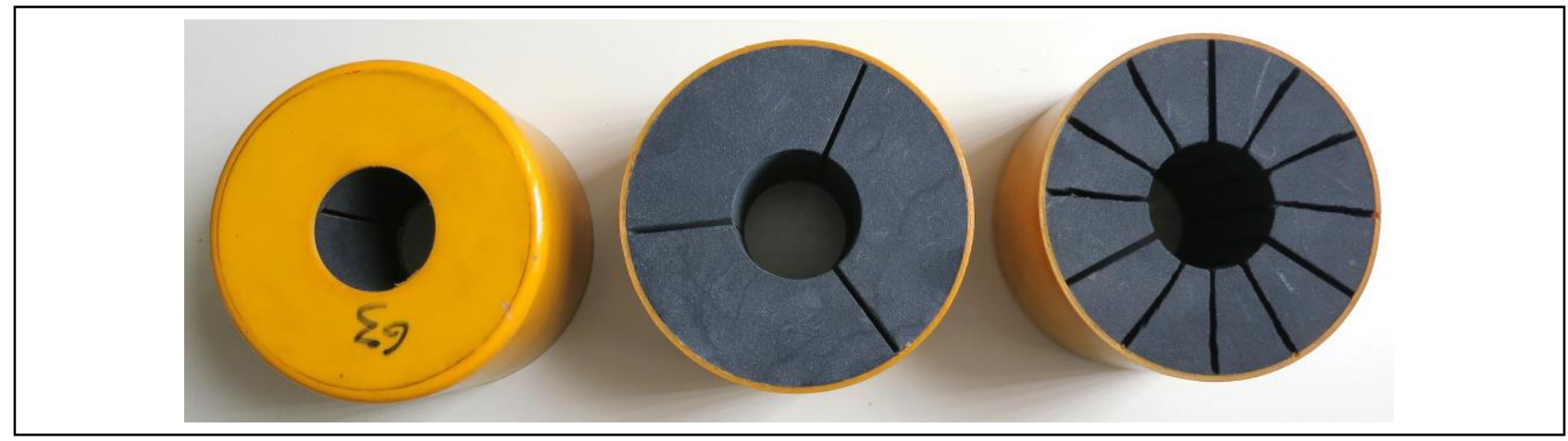

Fig. 3. Propellant grains for base bleed unit

Base drag reduction is modeled as function of nondimensional injection parameter I which is defined as:

$$
I=\frac{\dot{m}_{e}}{\rho_{\infty} V_{\infty} A_{b}}
$$

where: $\dot{m}_{e}$ is mass flow rate of combustion products bleeding through orifice, $\rho_{\infty}$ is local air density, $V_{\infty}$ is local air velocity and $A_{b}$ is area of projectile base.

\subsection{Base bleed propellant grain}

Propellant grain in the shape of apple with 2 segments with inner channel (Fig. 4.) will be studied with initial dimensions as following: $l_{0}$ is maximum length, $r_{0}$ is inner channel radius, $R$ is maximum radius, $r_{S}$ is radius of curved ends of grain and $w_{0}$ is half of distance between segments
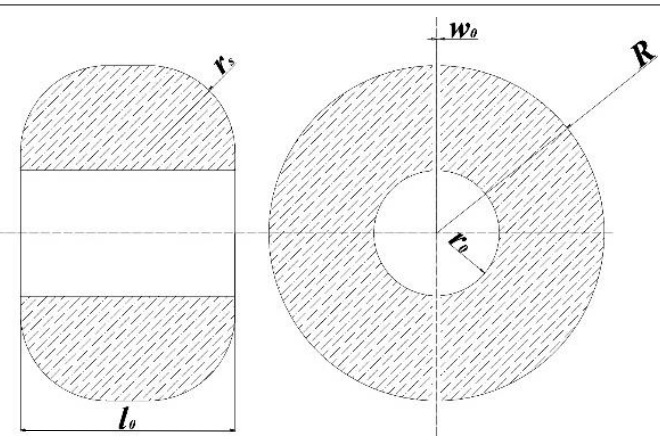

Fig. 4. Geometry of base bleed grain

Propellant grain is inhibited over outer surface, so the burning happens on the cylindrical surfaces of inner channel and flat surfaces of the slots. If propellant grain is exposed to the field of radial acceleration, which is case during the flight of artillery projectile with base bleed, burning rate will depend both on orientation of vector of acceleration relative to the burning surface and the level of radial acceleration. 
Noticeable increase of burning rate of propellant charge in the field of radial acceleration is happening on combustion surfaces which form angles of $75^{\circ}-90^{\circ}$ with the vector of acceleration (Anon, 1971).

During the grain burning in the field of radial acceleration, centrifugal force and tangential inertial force are acted on gas flow above the burning surface. Tangential component of inertial force has much smaller intensity than radial component of inertial force.

For propellant charge with shape shown in Fig. 4. gas flow is bending the flame towards cylindrical surface of grain and increase the burning rate on this surface because of more intense transfer of heat. This is happening because of centrifugal force. In the zone of slots, gas flow, by centrifugal force acting, is moving from the axis of symmetry to the wall of the chamber. Because of tangential component of inertial force, gas flow in slots is bending the flame towards one surface of the slot and pulls it away from the other surface. On the one surface of a slot flame is being bent to, transfer of heat is more intense and the burning rate is increasing. On the opposite surface flame is being bend away from, transfer of heat is reducing from gas flow to the surface of fuel and the burning rate is decreasing in comparison with the burning rate of propellant grain which is not exposed to the field of radial acceleration. So, the burning rate on cylindrical surface of inner channel $\dot{r}_{c}$ is different from the burning rate on flat surface of slot $\dot{r}_{p}$ when propellant grain is exposed to the field of radial acceleration.

Based on experiments (Kayser, et al., 1988), burning rates $\dot{r}_{c}$ and $\dot{r}_{p}$ can be shown in dependance of the burning rate $\dot{r}_{0}$ in static conditions, as:

$$
\begin{aligned}
& \dot{r}_{c}=f_{c}(p) \dot{r}_{0} \\
& \dot{r}_{p}=f_{p}(p) \dot{r}_{0}
\end{aligned}
$$

with functions $f_{c}(p)$ and $f_{p}(p)$ determined experimentally for the chosen propellant charge.

Velocity of combustion products generation is the regression rate of solid propellant, as shown in Fig. 5.

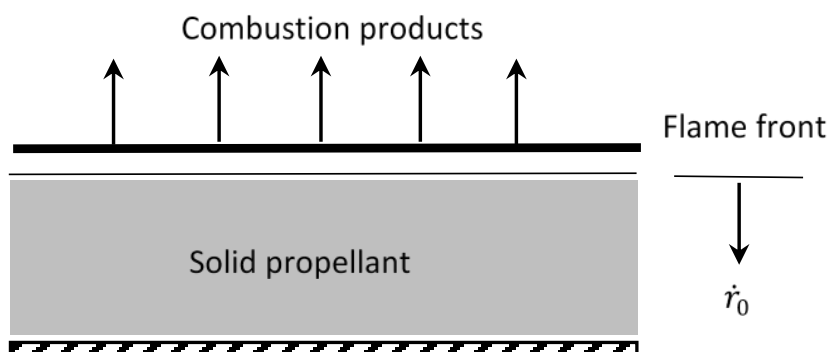

Fig. 5. Surface regression and gas combustion products generation

The burning rate is function of pressure and generally, in limited region of pressure, it is expressed by de Saint-Robert's law: 


$$
\dot{r}_{0}=a \cdot p^{n}
$$

where pressure exponent, $n$, and burn rate constant, $a$, are depended on chemical composition of propellant and initial temperature of propellant charge.

\subsection{The movement of flame front and instant area of burning surface}

During the combustion of propellant charge, shown in Fig. 2., which is exposed to the field of radial acceleration, the radius change of the inner cylinder at any instant is:

$$
r=\int_{r_{0}}^{r} \dot{r}_{c} d t
$$

and the regression of slots is:

$$
w=\int_{w_{0}}^{w} \dot{r}_{p} d t
$$

In first phase (Fig. 6.) front of the flame is proving from $r_{0}$ to $\left(R-r_{S}\right)$. Length of the grain in this phase is constant and is equal to the initial length of grain $l_{0}$.

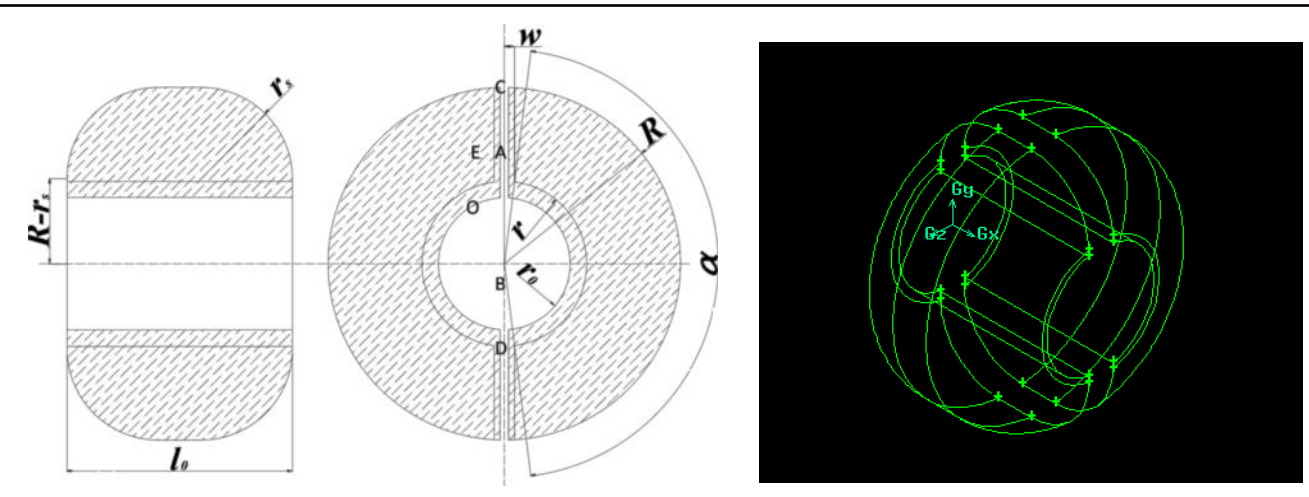

Fig. 6. First phase of flame front movement

The area of cylindrical surface $A_{c}$, at the moment $\mathrm{t}$, is equal to double product of length of arc $\widehat{A B}$ and length of the propellant charge $l_{0}$ :

$$
A_{c}=2 \cdot \widehat{A B} \cdot l_{0}=2 \cdot r \cdot \alpha \cdot l_{0}
$$

From Fig. 4. follows that:

$$
\begin{aligned}
& \sin \left(\frac{\pi}{2}-\frac{\alpha}{2}\right)=\frac{w}{r} \\
& \alpha=\pi-2 \cdot \arcsin \frac{w}{r} \\
& A_{c}=2 \cdot\left(\pi-2 \cdot \arcsin \frac{w}{r}\right) r \cdot l_{0}
\end{aligned}
$$


Area of slot $A_{p}$ at the moment $\mathrm{t}$ is:

$$
A_{p}=4 \cdot \overline{A C} \cdot l_{0}=4 \cdot\left(\sqrt{R^{2}-w^{2}}-\sqrt{r^{2}-w^{2}}\right) \cdot l_{0}
$$

Total area of combustion is:

$$
A_{s}=A_{c}+A_{p}
$$

and for $r \leq R-r_{S}$

$$
A_{s}=2 \cdot\left[\left(\pi-2 \cdot \arcsin \frac{w}{r}\right) r+2 \cdot\left(\sqrt{R^{2}-w^{2}}-\sqrt{r^{2}-w^{2}}\right)\right] \cdot l_{0}
$$

Second phase (Fig. 7.) covers movement of flame front for $r>R-r_{s}$.

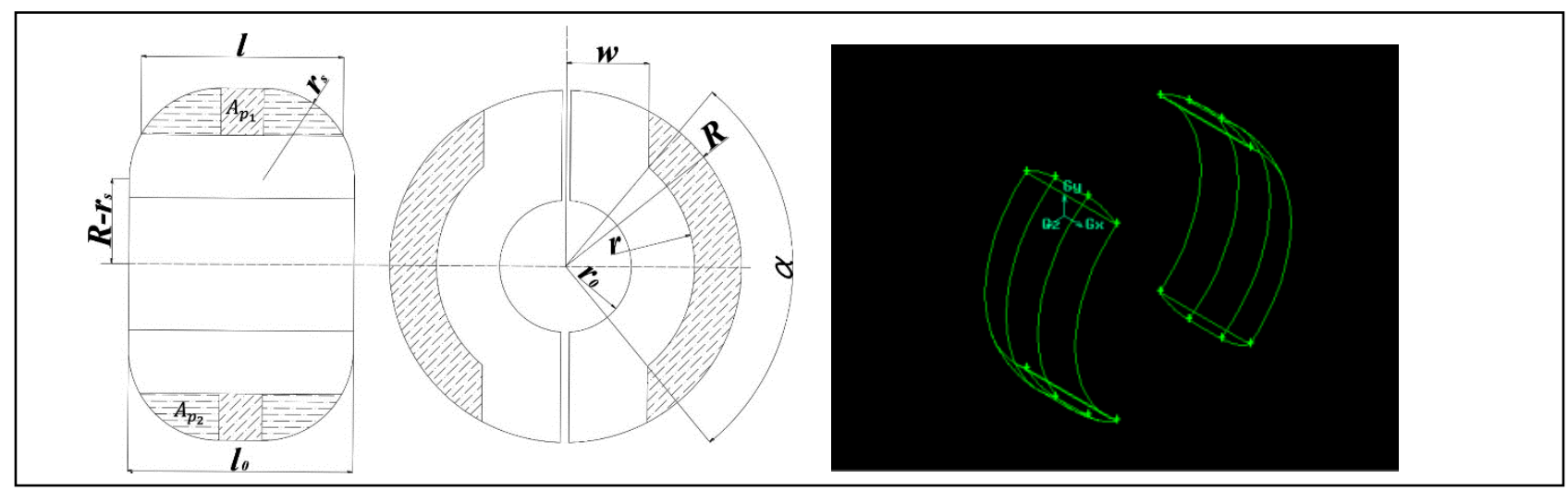

Fig. 7. Second phase of flame front movement

The area of cylindrical surface $A_{c}$, in some moment $\mathrm{t}$ in second phase is:

$$
A_{c}=2 \cdot r \cdot \alpha \cdot l
$$

Length of the grain in that moment $t$ is:

$$
l=l_{0}-2 \cdot\left[r_{s}-\sqrt{r_{s}^{2}-\left(r_{s}-R+r\right)^{2}}\right]
$$

so, considering (9), area of cylindrical surface in second phase is determined as:

$$
A_{c}=2 \cdot r \cdot\left(\pi-2 \cdot \arcsin \frac{w}{r}\right) \cdot\left\{l_{0}-2 \cdot\left[r_{s}-\sqrt{r_{s}^{2}-\left(r_{s}-R+r\right)^{2}}\right]\right\}
$$

Area of slot $A_{p}$ in moment t, according to Fig. 6., is:

$$
A_{p}=4 \cdot\left(A_{p_{1}}+2 \cdot A_{p_{2}}\right)
$$


where:

$$
\begin{aligned}
& A_{p_{1}}=\left(l_{0}-2 \cdot r_{s}\right)\left(\sqrt{R^{2}-w^{2}}-\sqrt{r^{2}-w^{2}}\right) \\
& A_{p_{2}}=\int_{\sqrt{r^{2}-w^{2}}}^{\sqrt{r^{2}}} \sqrt{r_{s}^{2}-\left(\sqrt{z^{2}+w^{2}}-R+r_{s}\right)^{2}} d z
\end{aligned}
$$

Total area of combustion for $r>R-r_{s}$ is:

$$
A_{s}=A_{c}+A_{p}=A_{c}+4 \cdot\left(A_{p_{1}}+2 \cdot A_{p_{2}}\right)
$$

where $A_{c}, A_{p_{1}}$ and $A_{p_{2}}$ are determined by (16), (18) and (19).

For propellant charge in the shape of apple with $\mathrm{n}$ segments with inner channel, total area of combustion is:

- In first phase

$$
A_{s}=2 \cdot\left[\left(\pi-2 \cdot n \cdot \arcsin \frac{w}{r}\right) r+2 \cdot n \cdot\left(\sqrt{R^{2}-w^{2}}-\sqrt{r^{2}-w^{2}}\right)\right] \cdot l_{0}
$$

- In second phase

$$
A_{s}=A_{c}+A_{p}=A_{c}+2 \cdot n \cdot\left(A_{p_{1}}+2 \cdot A_{p_{2}}\right)
$$

where:

$$
A_{c}=2 \cdot r \cdot\left(\pi-2 \cdot n \cdot \arcsin \frac{w}{r}\right) \cdot\left\{l_{0}-2 \cdot\left[r_{s}-\sqrt{r_{s}^{2}-\left(r_{s}-R+r\right)^{2}}\right]\right\}
$$

and areas $A_{p_{1}}$ and $A_{p_{2}}$ determined by (18) and (19).

\subsection{The mass flow rate of combustion products}

Mass flow rate generated by burning propellant is:

$$
\dot{m}=\rho_{p m}\left(\dot{r}_{c} \cdot A_{c}+\dot{r}_{p} \cdot A_{p}\right)
$$

i.e., with (2) and (3),

$$
\dot{m}=\rho_{p m}\left(f_{c}(p) \cdot A_{c}+f_{p}(p) \cdot A_{p}\right) \cdot \dot{r}_{0}
$$

During the bleed of combustion products through orifice with a sharp edge, where area of orifice is $A_{0}=\left(d_{e}^{2} \cdot \pi\right) / 4$, the flow is forced to converge to pass through the orifice. Contraction factor of flow $C_{c}$ presents ratio of cross area of flow and area of orifice. Cross area of flow A is because of that: 


$$
A=C_{c} \cdot A_{o}
$$

Real fluids are viscous so the part of mechanical energy on its travel through orifice and away transform to inner energy because of viscous forces, which means that mechanical energy (velocity) for the case of real fluid be smaller. That is considered with factor of correction of velocity $C_{v}$ which is determined experimentally.

Because of fluid viscosity, real velocity of flow $u_{e}$ is:

$$
u_{e}=C_{v} \cdot u
$$

where $u$ is the velocity of flow in orifice.

Volumetric flow rate $Q$ of products of combustion through the orifice will be equal to the product of real velocity and real area of flow:

$$
Q=u_{e} \cdot A=\underbrace{C_{v} \cdot C_{c}}_{C_{d}} \cdot u \cdot A_{o}
$$

where $C_{d}=C_{v} \cdot C_{c}$ is factor of correction of the flow.

Mass flow rate of combustion products through the orifice in the base of base bleed is:

$$
\dot{m}_{e}=\rho_{e} \cdot Q=\rho_{e} \cdot u_{e} \cdot A
$$

where $\rho_{e}$ is the density of combustion products at the exit.

Flow of combustion products from base bleed can be described with energy equation for incompressible flow - Bernoulli equation:

$$
p_{c}-p_{e}=\frac{\rho_{e} \cdot u^{2}}{2}
$$

i.e., taking correction of velocity caused by flow narrowing under consideration:

$$
p_{c}-p_{e}=\frac{1}{C_{v}^{2}} \cdot \frac{\rho_{e} \cdot u_{e}^{2}}{2}
$$

where $p_{e}$ is the pressure at the exit from base bleed. If Bernoulli equation is written as:

$$
p_{c}-p_{e}=\frac{1}{C_{v}^{2}} \cdot \frac{\rho_{e}^{2} \cdot u_{e}^{2}}{2 \cdot \rho_{e}} \cdot \frac{A^{2}}{A^{2}}
$$

taking under consideration equations (26) and (29), will be 


$$
p_{c}-p_{e}=\frac{1}{C_{v}^{2}} \cdot \frac{\dot{m}_{e}^{2}}{2 \cdot \rho_{e}} \cdot \frac{1}{C_{c}^{2} \cdot A_{o}^{2}}
$$

and mass flow rate of combustion products is:

$$
\dot{m}_{e}=\left(C_{d} \cdot A_{o} \cdot \sqrt{2 \cdot \rho_{e} \cdot p_{e}}\right) \sqrt{\frac{p_{c}}{p_{e}}-1}
$$

When the velocity of flow is higher than 0.3 Mach, the compressibility effect must be taken under consideration. We assume that the temperature of ideal gas is constant through streamline i.e., the flow is isothermal.

Because the combustion products are considered as ideal gas, the equation of state is:

$$
p=\rho \cdot R_{g} \cdot T_{c}
$$

where $R_{g}$ is the gas constant of combustion products and $T_{c}$ is the temperature of gas products of combustion and it is considered to be constant.

For steady, inviscid, isothermal flow, energy equation is:

$$
\int \frac{d p}{\rho}+\frac{1}{2} \cdot v^{2}=\text { const }
$$

Used on base bleed taking under consideration the equation of state $\left(\int \frac{d p}{\rho}=R_{g} \cdot T_{c} \int \frac{d p}{\rho}\right)$ :

$$
R_{g} \cdot T_{c} \cdot \ln p_{c}=R_{g} \cdot T_{c} \cdot \ln p_{e}+\frac{1}{2} \cdot u_{e}^{2}
$$

As $\frac{p_{c}-p_{e}}{p_{e}} \ll 1$ then $\ln \left(\frac{p_{c}}{p_{e}}\right)=\ln \left(1+\frac{p_{c}-p_{e}}{p_{e}}\right)=\frac{p_{c}}{p_{e}}-1$ and energy equation for compressible flow of combustion products in base bleed is:

$$
\frac{p_{c}}{p_{e}}-1=\frac{u_{e}^{2}}{R_{g} \cdot T_{c}}
$$

Velocity of combustion products through the orifice in the base of the projectile can be expressed by Mach number $M_{e}$ :

$$
u_{e}=M_{e} \cdot a
$$

where $a$ is velocity of sound in combustion products.

Considering that for isentropic flow: 


$$
\frac{p_{c}}{p_{e}}=\left(1+\frac{\gamma_{g}-1}{2} M_{e}^{2}\right)^{\frac{\gamma_{g}}{\gamma_{g}-1}}
$$

Mach number can be expressed as:

$$
M_{e}=\sqrt{\frac{2}{\gamma_{g}-1} \cdot\left[\left(\frac{p_{c}}{p_{e}}\right)^{\frac{\gamma_{g}-1}{\gamma_{g}}}-1\right]}
$$

and because,

$$
a=\sqrt{\gamma_{g} \cdot R_{g} \cdot T_{c}}
$$

with $\gamma_{g}$ being the relation of specific heats of products of combustion, velocity of flow at the exit is:

$$
u_{e}=\sqrt{\frac{2 \cdot \gamma_{g} \cdot R_{g} \cdot T_{c}}{\gamma_{g}-1} \cdot\left[\left(\frac{p_{c}}{p_{e}}\right)^{\frac{\gamma_{g}-1}{\gamma_{g}}}-1\right]}
$$

and

$$
\frac{p_{c}}{p_{e}}-1=\frac{\gamma_{g}}{\gamma_{g}-1} \cdot\left[\left(\frac{p_{c}}{p_{e}}\right)^{\frac{\gamma_{g}-1}{\gamma_{g}}}-1\right]
$$

So, taking compressibility under consideration, mass flow rate of bleeding of combustion products through the orifice of base bleed is:

$$
\dot{m}_{e}=\left(C_{d} \cdot A_{o} \cdot \sqrt{2 \cdot \rho_{e} \cdot p_{e}}\right) \sqrt{\frac{\gamma_{g}}{\gamma_{g}-1} \cdot\left[\left(\frac{p_{c}}{p_{e}}\right)^{\frac{\gamma_{g}-1}{\gamma_{g}}}-1\right]}
$$

At subsonic flow of gas, the pressure in the orifice through which the gas is bleeding is equal to the pressure of ambient the gas is bleeding into.

\subsection{A module for calculation of interior ballistics characteristics of base bleed}

A module for calculation of interior ballistics characteristics of base bleed enables the calculation of the mass flow rate at the exit of the base bleed as function of the time, for different grain configurations. This module is developed as a separate FORTRAN program IBBB and it can be integrated in program for trajectory of base bleed projectile estimating. 
Module is based on the following assumptions:

- Burn rate of propellant is independent of spin until the moment of establishing a quasi-steady mode

- Flow is one dimensional in the combustion chamber of base bleed

- Propellant burning is completely and adiabatic.

- Combustion products behave as ideal gas

- Pressure at base bleed exit is equal to ambient pressure (for the subsonic speed of injection)

Input file for program contains following data: geometrical characteristics of grain correcting for width of inhibitor, parameters for burning law of propellant, characteristics of propellant and combustion products, orifice diameter and spin rate of base bleed.

Exit file contains following data: time, chamber pressure and mass flow rate of combustion products at the exit of base bleed.

\section{Results}

Burning of base bleed propellant grain for projectile $155 \mathrm{~mm}$, M864, is simulated in static condition and in field of constant radial acceleration

In all cases of simulation, combustion products bleed in atmosphere. Time for establishing a quasi-steady mode is assumed to be 3.3 seconds. Inhibitor which coats the surfaces adjacent to the chamber case is taking into account by reduction of grain dimensions. Input data are shown in Tab. 1.

\begin{tabular}{|l|l|}
\hline Parameter & Value \\
\hline Number of segments & 2 \\
\hline Burn rate constant $(\mathrm{mm} / \mathrm{s}$ for pressure in bar) & 0.9132 \\
\hline Pressure exponent & 0.6655 \\
\hline Specific heat ratio of combustion products & 1.25 \\
\hline Length of grain $(\mathrm{mm})$ & 80.6 \\
\hline Maximum radius of grain $(\mathrm{mm})$ & 63.5 \\
\hline Radius of inner channel $(\mathrm{mm})$ & 24.15 \\
\hline Radius of curved ends of grain $(\mathrm{mm})$ & 31.5 \\
\hline Half of distance between segments $(\mathrm{mm})$ & 1.5 \\
\hline Web $(\mathrm{mm})$ & 39.35 \\
\hline Density of propellant $\left(\mathrm{kg} / \mathrm{m}^{3}\right)$ & 1532 \\
\hline Orifice diameter $(\mathrm{mm})$ & 48,3 \\
\hline Spin rate $(\mathrm{rev} / \mathrm{s})$ & $0-253$ \\
\hline
\end{tabular}

Tab. 1. Input data for FORTRAN program IBBB

Ignition of propellant charge is not considered. For the simulation of dynamic conditions, the selected values of spin rate are the same as values of experiments (Kayser et al., 1988) on the ground testing. Functions $f_{c}(p)$ and $f_{p}(p)$ determined experimentally for the chosen propellant charge are shown in Fig. 4. 
Fig. 8. shows change of pressure in combustion chamber as function of working time of base bleed at different constant spin rates those results of simulations.

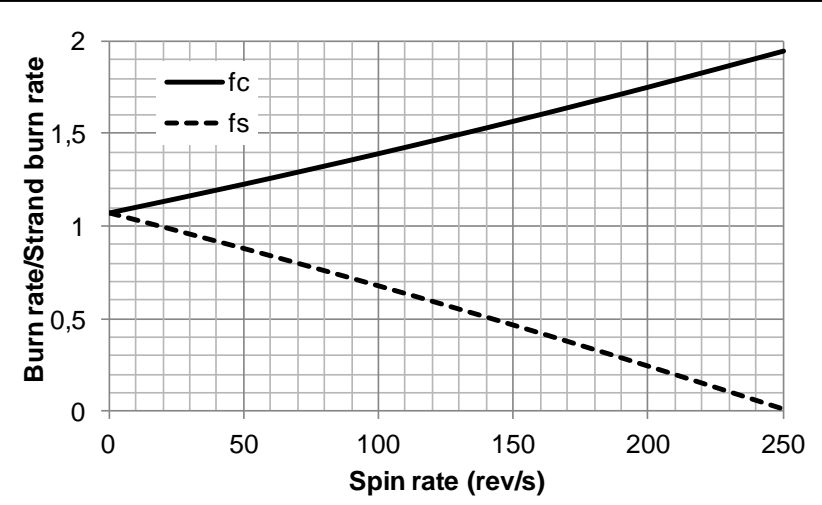

Fig. 8. Effect of spin rate on burn rate for the cylindrical and slot surfaces (SerdarevicKadic et al., 2012)

Results of experimental measurement (Danberg, 1990) are shown at Fig. 9., in the upper right corner. Pressure is expressed in atmospheres $(1 \mathrm{~atm}=101325 \mathrm{~Pa})$ to allow comparison with experimental results.

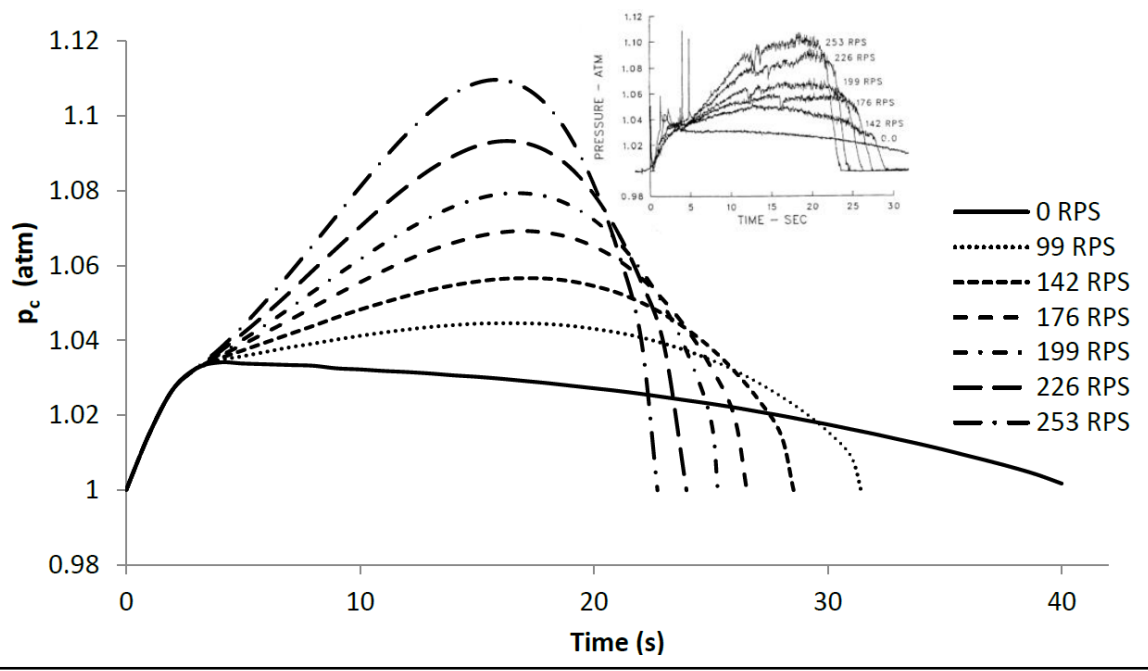

Fig. 9. Change of pressure in the combustion chamber of base bleed in the function of time with different constant spin rate

Propellant charge which combusts in static conditions has an increase of pressure in the initial phase that lasts around 3.3 seconds. After that, the pressure slightly decreases. When the propellant charge is exposed to a field of constant radial acceleration, combustion pressure continues to increase after the initial phase. In the ending phase (around $2 / 3$ of charge burning time), the combustion pressure starts to decrease rapidly.

Propellant charge of base bleed which is exposed to the field of constant radial acceleration combusts faster than the one in the static conditions. The burning rate depends on the level of acceleration and the position of acceleration vector in relation to the combustion surface. 
In the Tab. 2. are shown the combustion times of the base bleed (base bleed work), when the base bleed is exposed to the field of constant radial acceleration, in the function of spin rate used in experiments (Danberg, 1990) and simulation of combustion according to model for inner ballistic characteristics calculation of base bleed unit. By comparing the results of simulation (Fig. 9. and Tab. 2.) with the results of the experiment (Kayser et al., 1988), very good agreement is obtained.

\begin{tabular}{|r|c|c|c|}
\hline \multirow{2}{*}{ Spin rate (rev/s) } & \multicolumn{3}{|c|}{ Working time of base bleed (s) } \\
\cline { 2 - 4 } & $($ Danberg, 1990) & Results of simulation & Difference (\%) \\
\hline 0 & 40.0 & 39.93 & 0,175 \\
\hline 99 & 31.9 & 31.17 & 2,29 \\
\hline 142 & 29.0 & 28.14 & 2,96 \\
\hline 176 & 27.4 & 26.58 & 2,99 \\
\hline 199 & 26.0 & 24.82 & 4,54 \\
\hline 226 & 23.9 & 23.37 & 2,22 \\
\hline 253 & 22.8 & 22.46 & 1,5 \\
\hline
\end{tabular}

Tab. 2. Base bleed time of work with different spin rate

Effects of base bleed unit geometry at different spin rate is investigated. For appleshaped propellant charge with inner channel number of segments was changed from 2 to 12 and time of work is calculated for different spin rate (Tab. 3.).

\begin{tabular}{|r|r|r|r|r|r|r|}
\hline \multicolumn{7}{|c|}{ Working time (s) } \\
\hline \multirow{2}{*}{ Spin rate (rev/s) } & \multicolumn{7}{|c|}{ Number of segments } \\
\cline { 2 - 7 } & 2 & 3 & 4 & 6 & 8 & 12 \\
\hline 0 & 39.93 & 39.91 & 39.88 & 39.74 & 39.52 & 38.73 \\
\hline 99 & 31.17 & 31.2 & 31.23 & 31.27 & 31.28 & 31.21 \\
\hline 142 & 28.14 & 28.19 & 28.25 & 28.32 & 28.38 & 28.45 \\
\hline 176 & 26.58 & 26.64 & 26.7 & 26.8 & 26.88 & 27.02 \\
\hline 199 & 24.82 & 24.89 & 24.95 & 25.07 & 25.17 & 25.34 \\
\hline 226 & 23.37 & 23.44 & 23.51 & 23.63 & 23.74 & 23.93 \\
\hline 253 & 22.46 & 22.53 & 22.6 & 22.73 & 22.85 & 23.04 \\
\hline
\end{tabular}

Tab. 3. Effect of number of segments on working time for apple-shaped propellant charge with inner channel

Increasing number of segments at static condition is led to a slight reduction in work time of base bleed unit. At the maximum spin rate, increasing the number of segments from 2 to 12 is caused increasing of working time by $2.58 \%$.

For cylindrical propellant charge with inner channel is assumed that has the same maximum dimensions as apple-shaped propellant charge with inner channel. Number of segments and spin rate are changed and working time of the base bleed unit is calculated by program. The results are shown in table 4 . Increasing number of segments from 2 to 6 at static condition is led to a slight increasing in work time of base bleed unit. 
By further increasing the number of segments, working time is reduced because the surface of inner channel is significantly reduced. At the maximum spin rate, increasing the number of segments from 2 to 12 is caused increasing of working time by $4.26 \%$.

\begin{tabular}{|r|r|r|r|r|r|r|}
\hline \multicolumn{7}{|c|}{ Working time (s) } \\
\hline \multirow{2}{*}{ Spin rate (rev/s) } & \multicolumn{7}{|c|}{ Number of segments } \\
\cline { 2 - 7 } & 2 & 3 & 4 & 6 & 8 & 12 \\
\hline 0 & 40.11 & 40.3 & 40.38 & 40.41 & 40.22 & 39.01 \\
\hline 99 & 31.07 & 31.25 & 31.4 & 31.65 & 31.76 & 31.75 \\
\hline 142 & 27.92 & 28.1 & 28.25 & 28.5 & 28.68 & 28.86 \\
\hline 176 & 26.26 & 26.42 & 26.56 & 26.8 & 27 & 27.26 \\
\hline 199 & 24.4 & 24.56 & 24.7 & 24.95 & 25.15 & 25.45 \\
\hline 226 & 22.83 & 22.98 & 23.11 & 23.34 & 23.55 & 23.86 \\
\hline 253 & 21.82 & 21.94 & 22.05 & 22.26 & 22.44 & 22.75 \\
\hline
\end{tabular}

Tab. 4. Effect of number of segments on working time for cylinder-shaped propellant charge with inner channel

\section{Conclusion}

A model for estimation of inner ballistic characteristics of base bleed is established. The model allows the prediction of mass flow rate of combustion products at any moment of base bleed work. Modeling the mass flow rate is happening in the function of instant spin rate, surrounding atmosphere pressure and the temperature of combustion product. A model is verified by experimental data for base bleed grain of M864 projectile.

The analysis of the base bleed unit performance used conservation law of mass and conservation law of momentum, a burning rate law and the geometry of the solid propellant grain to predict the mass flow rate at the orifice of base bleed unit. The effect of spin rate on the burning rates of propellant grain is determined from experimental data. The burning surface is divided into a cylindrical and slot surface with different burning rates on each.

Calculation can be made for different shapes of propellant charge: cylindrical propellant charge with inner channel with multiple segments and the apple-shaped propellant charge with inner channel with multiple segments, which are inhibited over outer surface. Effect of segments number on working time is analysed for two shapes of grain with the same maximum external dimensions. Authors are observed that by increasing the number of segments at maximum angular velocity working time of base bleed unit is increased about one second.

One of the major model limitations is the timing of the ignition phase that is assumed constant for different spin rates and simulation of grain propellant ignition in real condition during the projectile launch is one direction of future research. 
Results of developed model enable estimation of mass flow rate at orifice of base bleed unit and therefore drag coefficient reduction. Future work will be implementation the model in the program for projectile trajectory prediction - modified mass point model (4DOF), with respect to projectile mass characteristics changing.

\section{References}

Anon. (1971). Solid rocket motor performance analysis and prediction, NASA Report SP 8039.

Belaidouni, H. Zivkovic, S. \& Samardzic, M. (2016). Numerical Simulations in Obtaining Drag Reduction for Projectile with Base Bleed. Available from: http://www. scindeks-clanci.ceon.rs Accessed: 2018-11-13

Danberg, J. E. (1990). Analysis of the flight performance of the $155 \mathrm{~mm}$ M864 base burn projectile. Report BRL-TR-3083, U.S. Army Ballistic Research Laboratory, Aberdeen Proving Ground, MD.

Gibeling, H.J. \& Buggeln, R. C. (1991). Reacting Flow Models for Navier-Stokes Analysis of Projectile Base Combustion. In: 27th Joint Propulsion Conference (AIAA / SAE / ASME / ASEE), Sacramento, CA.

Kaurinkoski P. (2000). Simulation of the Flow Past a Long-Range Artillery Projectile. $\mathrm{Ph}$. D. Dissertation, Helsinki University of Technology, Helsinki, Finland.

Kayser, L. D. Kuzan, J. D. \& Vazquez, D. N. (1988). Ground testing for base bleed systems. Report BRL-MR-3708, U.S. Army Ballistic Research Laboratory, Aberdeen Proving Ground, MD.

Nietubicz, C.J. \& Gibeling, H. J. (1995). Navier-Stokes Computations for a Reacting, M864 Base Bleed Projectile. Report ARL-TR-875, U.S. Army Ballistic Research Laboratory, Aberdeen Proving Ground, MD.

Serdarevic-Kadic, S.; Zecevic, B. Terzic, J. \& Catovic, A. (2012). Influence of local atmosphere characteristics to range of $155 \mathrm{~mm}$ M864 projectile. In: 15th Seminar New trends in research of energetic materials, part II, University of Pardubice, Czech Republic.

Zecevic, B.; Terzic, J. Razic, F. \& Serdarevic-Kadic, S. (2015). Lethal Influence Factors of Natural and Preformed Fragmentation Projectiles, Chapter 20 in DAAAM International Scientific Book 2015, pp. 219-234, B. Katalinic (Ed.), Published by DAAAM International, ISBN 978-3-902734-05-1, ISSN 1726-9687, Vienna, Austria 